\title{
Treatment of Dysphagia with Biofeedback and Functional Electrical Stimulation in a Patient with Wallenberg Syndrome: A Prospective Case Report
}

\author{
Daniela Jakobsen $^{\mathrm{a}}$ Rainer Seidl ${ }^{\mathrm{b}}$ Ingrid Poulsen ${ }^{\mathrm{a}, \mathrm{c}, \mathrm{d}}$ Derek John Curtis ${ }^{\mathrm{a}, \mathrm{e}}$ \\ aRUBRIC (Research Unit on Brain Injury Rehabilitation Copenhagen), Department of \\ Brain Injury, Copenhagen University Hospital, Copenhagen, Denmark; ${ }^{b}$ Department of \\ Otolaryngology at UKB, Hospital of the University of Berlin, Charite Medical School, Berlin, \\ Germany; 'Research Unit of Nursing and Health Care, Health, Aarhus University, Aarhus, \\ Denmark; ${ }^{d}$ Department of Clinical Research, Copenhagen University Hospital, Amager and \\ Hvidovre, Denmark; ${ }^{e}$ Child Centre Copenhagen, The Child and Youth Administration, City of \\ Copenhagen, Denmark
}

\section{Keywords}

Dysphagia - Wallenberg syndrome $\cdot$ Lateral medullary syndrome $\cdot$ Biofeedback · Functional electrical stimulation

\begin{abstract}
Biofeedback games and automated functional electrical stimulation (FES) can be used in the treatment of dysphagia. This case study aims to evaluate the effect of the treatment on a 77-year-old man with chronic Wallenberg syndrome and his and the therapist's experiences when using this therapy form. The participant received intensive treatment for nine days with Facial Oral Tract Therapy, biofeedback games and FES. The Penetration Aspiration Scale was scored using Functional Endoscopic Evaluation of Swallowing at baseline and the end of the intervention period. Swallowing-specific parameters were measured daily, and interviews were conducted with the patient and therapist during the intervention period. The patient and therapist both expressed a positive attitude to the ease of use and usefulness of this technology, despite there being no measurable change in the participant's swallowing and eating function and only small improvements in swallowing parameters. The experience from this study was that biofeedback games and FES gave only small improvements in swallowing for
\end{abstract}


Jakobsen et al.: Treatment of Dysphagia with Biofeedback and Functional Electrical Stimulation

this participant but were motivating and easy to use. Further research is needed to investigate the effect of this therapy on other participants with a more robust research design.

\section{Introduction}

Neurological insult affecting neuronal networks for swallowing in the brainstem can lead to severe dysphagia [1]. A reduced ability to eat and drink is associated with malnutrition, pneumonia, and increased mortality, social isolation, and anxiety [2].

Rehabilitation of dysphagia traditionally targets the re-organization and compensatory recruitment of swallowing-specific networks in the cerebral cortex [3]. For patients with neurogenic dysphagia, Coombes developed an interprofessional approach, the Facial Oral Tract Therapy ${ }^{\circledR}$ (F.O.T.T.) [4]. More recently, treatment for dysphagia has been supplemented with biofeedback and functional electrical stimulation (FES) [5]. Biofeedback aims to support learning and controlling movement patterns related to swallowing, eating, and drinking using visual feedback and has typically utilized surface electromyography (EMG) of the suprahyoid muscles to elevate the hyolaryngeal complex and thereby improve swallowing function. Some specialized equipment now combines these treatment modalities using surface EMG as a measure to trigger the FES of relevant surface muscles, supporting the subject's attempt to swallow. Biofeedback might help patients to modify their sensorimotor swallowing pattern since they suffer reduced intrinsic sensory feedback due to their neural lesion. A recent meta-analysis has shown it to have a positive effect on maximum displacement of the hyoid bone, but was inconclusive concerning the functional, physiological- or clinical outcome due to the poor quality and heterogeneity of the included studies [5]. A systematic review and meta-analysis from 2016 reported that treatment of swallowing function with FES seemed to be more effective than without in poststroke dysphagia in the short term [6].

In this prospective case report, we wanted to measure whether combined FES and biofeedback games could assist a patient with chronic dysphagia to reach his own selfformulated goals and at the same time investigate the experiences of the patient and the therapist when using this technology. The report was prepared in accordance with the CARE guidelines.

\section{Case Report/Case Presentation}

This was a prospective, single-case report examining the use, effect, and subjective experiences when using physiologically triggered FES in the treatment of swallowing function for a patient with chronic dysphagia in an outpatient setting.

\section{The Participant}

S.G. is a 77-year-old pensioner who was independent in his daily life despite a discrete left hemiparesis from earlier mild apoplexies. In 2013, an infarction in the cerebellum and the medulla oblongata, diagnosed as Wallenberg syndrome (WS), led to severe dysphagia. S.G.'s cognition was not affected, but he suffered a mild right side hemiparesis and persisting dysphagia, requiring percutaneous endoscopic gastrostomy. Rehabilitation as an inpatient, in municipal treatment centres, and as an outpatient had no lasting effect on S.G.'s dysphagia.

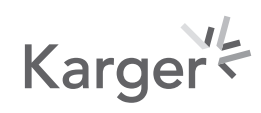


Today S.G. can swallow, but there is only minimal bolus transport into the oesophagus with a risk of aspiration. However, S.G. can protect his airways by hawking and spitting saliva out or coughing. He has not had pneumonia since his discharge from the hospital.

S.G. feels uncomfortable and socially embarrassed as he is unable to eat and drink with others and sometimes has to hawk and spit during a conversation. He does not join his family in restaurants, as he does not want to hawk and spit into a flask in public.

\section{Clinical Examination}

S.G. walks independently but insecurely due to problems with balance and strength. In sitting, S.G. has a malalignment with a posteriorly tilted pelvis and increased thoracic kyphosis with his neck ventrally translated. He does not swallow spontaneously but clears his pharynx and spits out every two-five min.

S.G. has a right-sided vocal cord and soft palate paresis (determined by laryngoscopy). During tactile examination of the mouth, he swallows regularly with initial pumping jaw movements. After three-four swallows, he clears his pharynx and spits.

\section{Method}

S.G. agreed 2 SMART (Specific, Measurable, Achievable, Relevant and Timebound) goals with the therapist prior to the intervention: to be able to drink 10 sips of thickened juice without spitting out and to spit out only once in a 5-min conversation. A clinical examination was carried out at baseline and at the end of the nine-day intervention using FEES (Fiberoptic Evaluation of Swallowing) to assess swallowing function using the Penetration Aspiration Scale (PAS). No solid consistencies or amounts of liquid or apple sauce larger than one teaspoon were used in the assessment to avoid aspiration. The FEES videos were scored by a highly experienced otolaryngologist who was blinded. Swallow-specific parameters were measured daily using the RehaIngest apparatus during F.O.T.T. in S.G.'s habitual sitting position as well as a sitting position and side-lying using the principles for Positioning in neutral (LIN) [4].

S.G. and the therapist were interviewed using a semistructured interview guide on days one, five and ten. The interviews for S.G. focused on quality of life, hopes and expectations and experiences with the intervention. The therapist was asked about the effectiveness and ease of use of the apparatus in the treatment. Interviews were conducted and recorded by one of the authors (I.P.) and transcribed. A deductive approach was used to analyse the data, according to the themes of the interviews.

\section{Description of Intervention}

The therapy comprised nine 60-min interventions over a two-week period. Electrical stimulation and biofeedback games were combined with F.O.T.T..

The training and measurement apparatus comprised RehaIngest that measures EMG and bioimpedance via surface electrodes and RehaMove, an FES system. RehaIngest provides biofeedback, measures swallow-specific parameters (laryngeal elevation, velocity and duration), and triggers RehaMove to stimulate the suprahyoidal muscles for swallowing. The biofeedback games train the timing and strength of swallowing.

This was followed by the training of swallowing in an aligned sitting position using biofeedback games and electrical stimulation. Fixation of the stimulation electrodes under the jaw was modified with a self-adhesive bandage for better contact. Training was divided into blocks of 15 swallows with short breaks to mobilize S.G. A patient-orientated protocol for EMG biofeedback, inspired by Steele et al. [7], was used. The intensity of electrical stimulation was adjusted daily to an effective but non-painful level and the F.O.T.T. intervention adapted.

\section{Karger'}


Case Reports in

Neurology

Table 1. PAS scores before and after the 9-day intervention

\begin{tabular}{l|ll}
\hline Case Rep Neurol 2021;13:789-796 & \\
\hline DOI: 10.1159/000518910 & $\begin{array}{l}\odot \text { 2 2021 The Author(s). Published by S. Karger AG, Basel } \\
\text { www.karger.com/crn }\end{array}$ & \\
\hline $\begin{array}{l}\text { Jakobsen et al.: Treatment of Dysphagia with Biofeedback and Functional Electrical } \\
\text { Stimulation }\end{array}$ & PAS score & \\
\hline Consistency & before & after \\
\hline & 2 & 2 \\
Cold, lightly thickened juice on teaspoon & 4 & 3 \\
Cold, moderately thickened juice on teaspoon & 2 & 4 \\
Cold, non-thickened juice on teaspoon & 2 & 2 \\
\hline
\end{tabular}

Values are the highest (worst) PAS scores of the swallows measured. PAS, Penetration Aspiration Scale (higher score is poorer).

Swallow training was performed by either chewing small pieces of apple in gauze or swallowing small boluses of maximum one teaspoon. The interventions were closely monitored to ensure that S.G. could protect his airways. The Mendelsohn manoeuvre [8] was also used in conjunction with the biofeedback games.

\section{Results}

\section{Measures of Swallowing Function}

S.G.'s swallowing function did not change during the intervention and neither of the SMART goals were achieved. There were, however, changes in the swallow-specific parameters, reflecting an improvement in the quality of swallowing towards a more normal pattern of movement.

\section{Endoscopic Evaluation}

Results from the PAS scores of the endoscopic evaluations are shown in Table 1 and demonstrate a lower (improved) PAS score for moderately thickened juice and a higher final PAS score for non-thickened juice.

\section{Swallow-Specific Parameters (Laryngeal Lift, Speed and Duration of Elevation)}

The swallow-specific parameters are presented in Figure 1. In lying, values are low compared to the published normal values and are unchanged over time. In sitting, maximum speed and elevation show no clear tendencies over time. Swallow duration in sitting appears to increase systematically during the intervention period.

\section{Patient and Therapist Experiences}

In terms of usefulness, S.G. felt some effect of the therapy, but ended on a score of 1-2 on a Likert scale where normal swallowing is a score of 10 . He did however express that after the intervention, he felt safe when the bolus travelled further into the oesophagus.

He found the intervention challenging, motivating, and tiring, but never unpleasant. He said that he would recommend the therapy to others with the same symptoms.

The therapist observed that the combination of F.O.T.T., FES and biofeedback games increased swallowing frequency and improved some of the swallow-specific parameters. S.G. developed a more coordinated swallowing pattern, with several swallow attempts for each bolus before hawking. But her hope that S.G. would be able to drink something, and experience it as drinking, was not fulfilled.

The therapist felt that using the apparatus required some experience, and it was threefour days before she was efficient at applying the electrodes. The biofeedback reflected her observations of the quality and pattern of S.G.'s swallows although sometimes the apparatus 


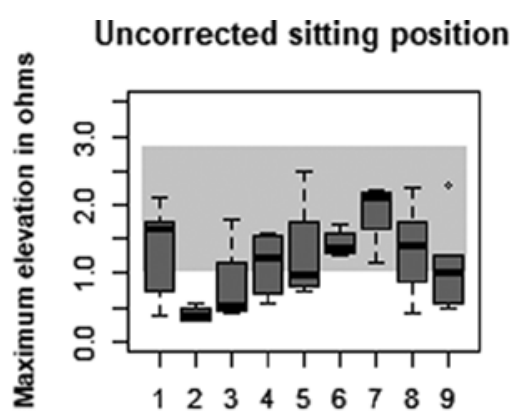

Corrected sitting position
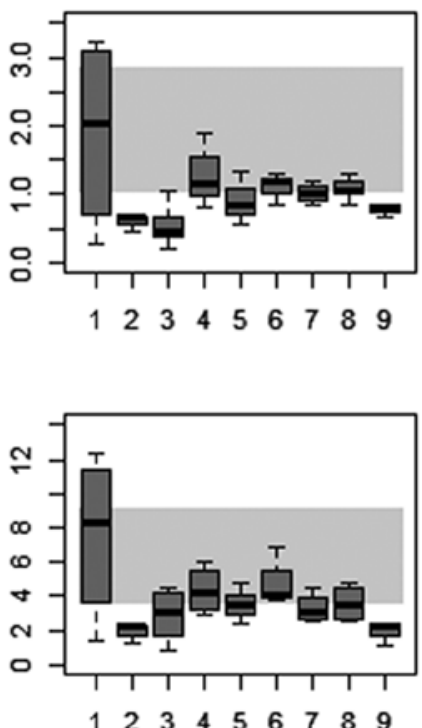

$\begin{array}{lllllllll}1 & 2 & 3 & 4 & 5 & 6 & 7 & 8 & 9\end{array}$

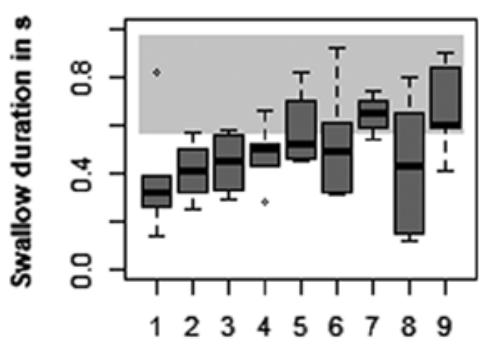

Day number

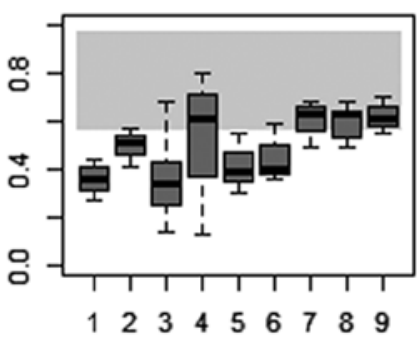

Day number
Lying position
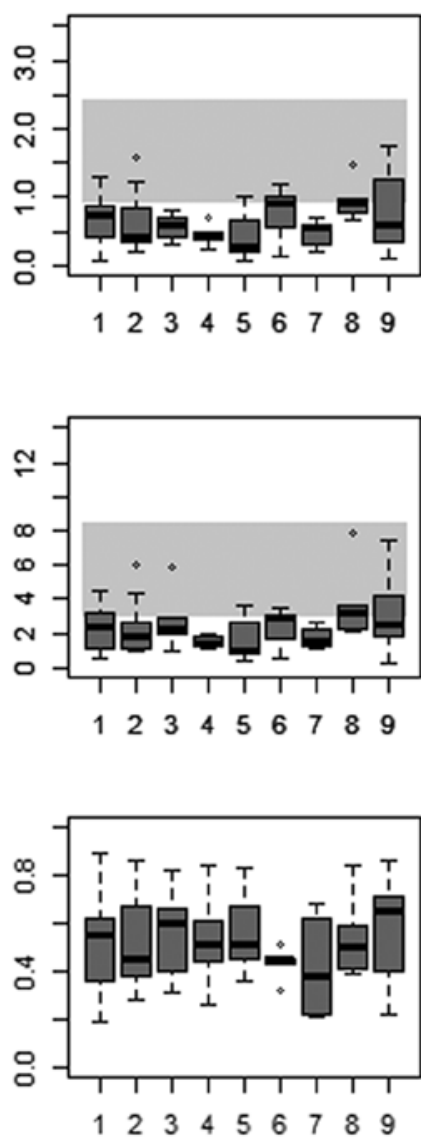

Day number

Fig. 1. Swallow-specific parameters during the 9 days of the intervention. Grey bands are mean \pm 1 SD for healthy subjects $[12,15]$. Normal values for swallow duration in lying for healthy subjects are not published.

did not record a swallow or falsely recorded a non-existent swallow. The therapist found it important to select, monitor and graduate the intervention to prevent aspiration and negative sensorimotor feedback.

She found determining the FES intensity and electrode placement challenging and suspected that the stimulation intensity had been too low initially despite S.G.'s very comprehensive feedback. She reflected that the important prerequisites for this therapy are that the patient can sit upright and can concentrate for 30 min. Furthermore, the patient needs to understand the situation and be able to use visual cues for initiating and modifying swallowing. No adverse effects, such as clinical signs of pneumonia, increased secretions in the upper or lower airways or episodes of coughing indicating aspiration, were observed during the intervention period.

\section{Discussion}

The lack of functional improvement was not unexpected in the light of a recent review and meta-analysis [5] of the effect of biofeedback on dysphagia, in which significant changes were reported in hyoid displacement but not in eating ability or feeding tube removal. In 
Jakobsen et al.: Treatment of Dysphagia with Biofeedback and Functional Electrical Stimulation

our case, the lack of functional improvement might be explained by the aetiology of S.G.'s dysphagia. In patients with WS, risk factors for aspiration are dysphonia, soft palate dysfunction and decreased facial sensitivity [9]. Six years after debut, S.G. still showed dysphonia and soft palate paresis. It may also be explained by the short intervention period. There is inconsistent evidence for patients with WS in the chronic phase regarding remittance of dysphagia [10].

Avoiding fatigue whilst maintaining the highest possible intensity to promote motor learning was challenging. Evidence of a positive dose-response relationship supports a high-intensity approach [11]. However, muscular or cognitive fatigue might prevent motor learning. To try and balance intensity and fatigue, we gave S.G. regular breaks during therapy.

Data from the swallow-specific parameters fluctuated greatly from day to day. The intrarater reliability of the measurement method is high [12]; therefore, the data most likely reflect S.G.'s variable performance. Swallow duration increased towards normality during the intervention period. The considerable fluctuations between the days show that S.G. has not reached a stable end point in his swallowing function and that the duration of the intervention might not be sufficient to stabilize the therapy results. Clinically, the swallowing pattern became more coordinated. S.G. verified this, when he reported that the bolus travelled further into the oesophagus.

We were unfortunately unable to verify S.G.'s experiences as we did not have the opportunity to carry out a videofluoroscopic evaluation of swallowing. This would have been useful in assessing the effectiveness of S.G.'s swallows more objectively than using endoscopy.

Placing the FES electrodes and determining the stimulation intensity was challenging. S.G.'s cooperation was necessary for a symmetric electrical stimulation without discomfort. A few times the apparatus stimulated, even though S.G. did not initiate a swallow. Falsepositive stimulation can lead to a behaviour in which the subject attempts to prevent inappropriate stimulation [13] and can lead to poor sensorimotor learning. The algorithms for swallowing detection are in a continuous process of improvement based on the results of this and other studies to reduce detection error.

One of the important aims of this study was to investigate whether this technology was likely to be accepted in clinical practice. Both S.G. and the therapist were positive about the ease of use and usefulness of this technology. S.G. experienced the biofeedback as motivating and challenging. A similar finding has been reported in another study targeting dysphagia [14] and also in other populations using biofeedback and FES with other treatment objectives.

It would appear that both the therapist and patient are positive concerning the potential benefits of the treatment in spite of the limited functional gain in this short intervention. If further trials with the apparatus show that this technology is useful in promoting functional changes in swallowing, then it is possible that it may be incorporated in routine clinical practice treating patients with the required sensory-motor, perceptive and cognitive resources.

\section{Statement of Ethics}

This study was conducted in accordance with the World Medical Association Declaration of Helsinki. The study protocol was submitted to and approved by the local ethics committee on human research (Videnskabsetisk Komité Region Hovedstaden Journal No. H-18030894). Written informed consent was obtained from the participant before the start of the study, and further written consent was obtained to publish this case.

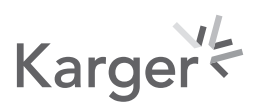




\section{Conflict of Interest Statement}

R.S. has been significantly involved in the development of RehaIngest. Travel and accommodation to Copenhagen in connection with the study were paid for by HASOMED GmbH. The authors have no other conflicts of interest to declare.

\section{Funding Sources}

This study was funded by the Helene Elisabeth Bunkelmann Marckwards Fund. This fund had no role in the preparation of data or the manuscript.

\section{Author Contributions}

All authors made substantial contributions to the conception and design of the work. D.J., D.J.C., and I.P. acquired the data, and all authors interpreted the data. All authors were involved in drafting and revising the work and giving final approval of the final version for publication and agree to be accountable for all aspects of the work in ensuring that questions related to the accuracy or integrity of any part of the work are appropriately investigated and resolved.

\section{Data Availability Statement}

The data that support the findings of this study cannot be shared due to local data protection regulations. Queries regarding the data in this article should be addressed to the corresponding author D.J.

\section{References}

1 Jean A. Brain stem control of swallowing: neuronal network and cellular mechanisms. Physiol Rev. 2001 Apr; 81(2):929-69.

2 Kjaersgaard A, Kristensen HK, Kjaersgaard A, Kristensen HK. Brain injury and severe eating difficulties at admission—patient perspective nine to fifteen months after discharge: a Pilot Study. Brain Sci. 2017 Aug 7;7: 96. http://www.mdpi.com/2076-3425/7/8/96.

3 Bath PM, Lee HS, Everton LF. Swallowing therapy for dysphagia in acute and subacute stroke. Cochrane Database Syst Rev. 2018 Oct 30;10(10):CD000323.

4 Jakobsen D, Poulsen I, Schultheiss C, Riberholt CG, Curtis DJ, Petersen TH, et al. The effect of intensified nonverbal facilitation of swallowing on dysphagia after severe acquired brain injury: a randomised controlled pilot study. NeuroRehabilitation. 2019;45:525-36.

5 Benfield JK, Everton LF, Bath PM, England TJ. Does therapy with biofeedback improve swallowing in adults with dysphagia? a systematic review and meta-analysis. Arch Phys Med Rehabil. 2019;100:551-61.

6 Chen YW, Chang KH, Chen HC, Liang WM, Wang YH, Lin YN. The effects of surface neuromuscular electrical stimulation on post-stroke dysphagia: a systemic review and meta-analysis. Clin Rehabil. 2016 Jan;30: 24-35.

7 Steele CM, Bennett JW, Chapman-Jay S, Polacco REC, Molfenter SM, Oshalla M. Electromyography as a biofeedback tool for rehabilitating swallowing muscle function. Applications of EMG in clinical and sports medicine. InTech; 2012.

8 Inamoto Y, Saitoh E, Ito Y, Kagaya H, Aoyagi Y, Shibata S, et al. The mendelsohn maneuver and its effects on swallowing: kinematic analysis in three dimensions using dynamic area detector CT. Dysphagia. 2018;33(4): 419-30.

9 Kim H, Chung CS, Lee KH, Robbins JA. Aspiration subsequent to a pure medullary infarction: Lesion sites, clinical variables, and outcome. Arch Neurol. 2000;57(4):478-83.

10 Teasell R, Foley N, Fisher J, Finestone H. The incidence, management, and complications of dysphagia in patients with medullary strokes admitted to a rehabilitation unit. Dysphagia. 2002;17:115-20. 
11 Lohse KR, Lang CE, Boyd LA. Is more better? using metadata to explore dose-response relationships in stroke rehabilitation. Stroke. 2014;45:2053-8.

12 Schultheiss C, Schauer T, Nahrstaedt H, Seidl RO. Evaluation of an EMG bioimpedance measurement system for recording and analysing the pharyngeal phase of swallowing. Eur Arch Otorhinolaryngol. 2013;270:214956.

13 Riebold B, Nahrstaedt H, Schauer T, Seidl RO. Self-adapting classification system for swallow intention detection in dysphagia therapy. Curr Dir Biomed Eng. 2019 Sep;5(1):49-52.

14 Dias P, Silva R, Amorim P, Laíns J, Roque E, Serôdio I, et al. Using virtual reality to increase motivation in poststroke rehabilitation: VR therapeutic mini-games help in poststroke recovery. IEEE Comput Graph Appl. 2019 Jan;39:64-70.

15 Schultheiss C, Wolter S, Schauer T, Nahrstaedt H, Seidl RO. [Effect of body position on coordination of breathing and swallowing]. HNO. 2015;63:439-46. 